PROCEEDINGS OF THE

AMERICAN MATHEMATICAL SOCIETY

Volume 128, Number 3, Pages 781-788

$\mathrm{S}$ 0002-9939(99)05064-9

Article electronically published on March 3, 1999

\title{
LINEAR EQUATIONS IN SUBSPACES OF OPERATORS
}

\author{
R. L. MOORE AND T. T. TRENT \\ (Communicated by David R. Larson)
}

\begin{abstract}
Given a subspace $\mathcal{S}$ of operators on a Hilbert space, and given two operators $X$ and $Y$ (not necessarily in $\mathcal{S}$ ), when can we be certain that there is an operator $A$ in $\mathcal{S}$ such that $A X=Y$ ? If there is one, can we find some bound for its norm? These questions are the subject of a number of papers, some by the present authors, and mostly restricted to the case where $\mathcal{S}$ is a reflexive algebra. In this paper, we relate the broader question involving operator subspaces to the question about reflexive algebras, and we examine a new method of forming counterexamples, which simplifies certain constructions and answers an unresolved question. In particular, there is a simple set of conditions that are necessary for the existence of a solution in the reflexive algebra case; we show that - even in the case where the co-rank of $X$ is one-these conditions are not in general sufficient.
\end{abstract}

In a sequence of papers ([2], [3], [4] ) the authors have studied the possibility of solving the operator equation $A X=Y$, where $X$ and $Y$ are given Hilbert space operators and $A$ is an operator to be found. In those papers, the operator $A$ is required to lie in the algebra associated with some commutative subspace lattice. Certain necessary conditions were derived in [3] for the existence of such an operator, and, in [4], we showed that those conditions are not sufficient. The construction of the counterexample in [4] was somewhat complicated, and the structure of the algebra studied there is obscure. In this paper we suggest a new way to construct counterexamples, which - on the one hand - connects the problems of finding unknown operators in CSL algebras with the problem of finding them in other operator subspaces, and - on the other - allows us to answer some unresolved questions about the sufficiency of the conditions derived in [3].

Throughout, $\mathcal{H}$ will represent a separable Hilbert space with inner product $\langle\cdot, \cdot\rangle$; $\mathcal{H}$ will sometimes be finite-dimensional. A subspace is a closed linear manifold, and we will often identify the subspace with the orthogonal projection whose range it is. If $\mathcal{L}$ is a collection of projections (or subspaces) that is closed under the operations of meet and join, it is a lattice. If, in addition, it contains the zero and identity operators, and is closed in the strong operator topology, it is called a subspace lattice. If the projections in $\mathcal{L}$ all commute with each other, then $\mathcal{L}$ is a commutative subspace lattice, or CSL. The collection of operators that leave invariant all the projections in $\mathcal{L}$ is called $\operatorname{Alg} \mathcal{L}$, and it is easy to see that $\operatorname{Alg} \mathcal{L}$ is a weakly closed algebra of operators. Standard terminology refers to the collection $\operatorname{Alg} \mathcal{L}$ as a reflexive algebra, and - if $\mathcal{L}$ is a CSL - as a CSL algebra.

Received by the editors April 22, 1998.

1991 Mathematics Subject Classification. Primary 47D25.

(C)1999 American Mathematical Society 
Let $N$ be either a positive integer or $\infty$. We adopt the notation $\mathbb{N}_{N}$ for the full set of natural numbers (if $N=\infty$ ) or the initial segment $\{1,2,3, \ldots, N\}$ (if $N<\infty$ ). Let $\mathcal{H}$ be a Hilbert space, and let $\left\{e_{n}\right\}_{n=1}^{N}$ be a fixed orthonormal basis - where, again, $N$ may be a positive integer or $\infty$ - and let $J$ be a subset of $\mathbb{N}_{N} \times \mathbb{N}_{N}$. The collection of operators $\mathcal{S}(J)=\left\{A \in B(\mathcal{H}):\left\langle A e_{k}, e_{i}\right\rangle=0\right.$ whenever $\left.(i, k) \notin J\right\}$ is a weakly closed linear subspace of $B(\mathcal{H})$. To put it another way: Write the matrix for an operator $A$ in the fixed basis; if the non-zero entries of the matrix are confined to the locations prescribed by the collection $J$, then $A$ is in the subspace $\mathcal{S}(J)$. So, the subspace $\mathcal{S}(J)$ is defined by its pattern of 0's in the given basis, and we refer to such subspaces as pattern subspaces. Only for certain choices of $J$ will $\mathcal{S}(J)$ be an algebra. For example, if $J=\{(i, k): i=k\}$, then $\mathcal{S}(J)$ is the algebra of diagonal operators. On the other hand, if $J=\{(i, k): i \neq k\}$, then $\mathcal{S}(J)$ is the subspace of operators with diagonal entries all zero, and is not an algebra. While not every pattern subspace is an algebra, any CSL algebra acting on a finite-dimensional space can be realized as an operator subspace of this kind. Furthermore, any pattern subspace which is an algebra - and which contains the diagonal operators - must be a CSL algebra.

In earlier papers, we have been interested in solving the operator equation $A X=$ $Y$, where $X$ and $Y$ are given operators, $\mathcal{L}$ is a fixed CSL, and $A$ is required to lie in the algebra $A l g \mathcal{L}$. It would be perfectly legitimate to ask for solutions of $A X=Y$ not just in reflexive algebras, but in pattern subspaces, and this we expect to do in a later article. For now, we observe that a standard trick will produce an algebra from any pattern subspace $\mathcal{S}(J)$. Let $\mathcal{D}$ represent the collection of diagonal operators in the basis $\left\{e_{n}\right\}$ and note that any pattern subspace (defined in the same basis, of course) is a bimodule over the algebra $\mathcal{D}$. Let $\mathcal{K}=\mathcal{H} \oplus \mathcal{H}$ and consider the collection of matrices formed as follows:

$$
\left[\begin{array}{cc}
D_{1} & S \\
0 & D_{2}
\end{array}\right]
$$

where $D_{1}$ and $D_{2}$ lie in $\mathcal{D}$ and $S$ must lie in $\mathcal{S}(J)$. It is easy to see that this collection is a weakly closed algebra and that it is also a pattern subspace containing the diagonal operators. Hence, it is a CSL algebra, and we refer to it as $A(J)$. These algebras are the subject of this paper, so we want to investigate the lattice of invariant subspaces more fully. Specifically, given a subset $J$ of $\mathbb{N}_{N} \times \mathbb{N}_{N}$, what is the lattice of invariant subspaces? Since the algebra $A(J)$ contains the set of operators that are diagonal in the basis $\left\{e_{1} \oplus 0, e_{2} \oplus 0, \ldots, 0 \oplus e_{1}, 0 \oplus e_{2}, \ldots\right\}$ in $\mathcal{K}$, any projection invariant under all the operators in $A(J)$ must itself be diagonal in this basis. Let $P$ and $Q$ be diagonal projections acting on $\mathcal{H}$ and suppose that $P \oplus Q$ is invariant for all operators in $A(J)$. We then have

$$
\left[\begin{array}{cc}
P^{\perp} & 0 \\
0 & Q^{\perp}
\end{array}\right]\left[\begin{array}{cc}
D_{1} & S \\
0 & D_{2}
\end{array}\right]\left[\begin{array}{cc}
P & 0 \\
0 & Q
\end{array}\right]=\left[\begin{array}{ll}
0 & 0 \\
0 & 0
\end{array}\right]
$$

which reduces to the equation $P^{\perp} S Q=0$ for all $S \in \mathcal{S}(J)$. Now let $e_{i} \otimes e_{k}^{*}$ represent the rank-one operator defined by $\left(e_{i} \otimes e_{k}^{*}\right) f=\left\langle f, e_{k}\right\rangle e_{i}$. Matricially, $e_{i} \otimes e_{k}^{*}$ is the matrix with a 1 in the $i$-kth position. Whenever $(i, k) \in J$, then $e_{i} \otimes e_{k}^{*} \in \mathcal{S}(J)$, and we have $0=P^{\perp}\left(e_{i} \otimes e_{k}^{*}\right) Q=\left(P^{\perp} e_{i}\right) \otimes\left(Q e_{k}\right)^{*}$. Therefore, whenever $(i, k) \in J$, we have either $e_{i} \in P$ or $e_{k} \in Q^{\perp}$.

Observe that if $P^{\perp} S Q=0$ for all $S \in \mathcal{S}(J)$, and if $P_{1} \geq P$, then $P_{1}{ }^{\perp} S Q=0$ as well. Therefore, if $Q$ is a diagonal projection, it will suffice to identify the 
minimal $P$ for which $P \oplus Q \in \operatorname{Lat}(A(J))$. Suppose $P \oplus Q$ is invariant for $A(J)$ and that $Q$ is the span of the basis vectors $\left\{e_{k}: k \in K\right\}$, and $P$ is the span of $\left\{e_{i}: i \in I\right\}$. By the last line of the previous paragraph, if $(i, k) \in J$, then either $i \in I$ or $k \notin K$. If $k \in K$, then, for each $i$ such that $(i, k) \in J$, we must have $i \in I$. For a given value of $k$, let $J_{k}$ represent the $k$-th cross-section of $J$, that is, $J_{k}=\{i:(i, k) \in J\}$. For each $k$, we have just argued that $J_{k} \subseteq I$. Therefore, we must have $\bigcup\left\{J_{k}: k \in K\right\} \subseteq I$. We summarize with a theorem.

Theorem. Let $\left\{e_{i}\right\}_{i=1}^{N}$ be a basis for $\mathcal{H}$, possibly with $N=\infty$. Let $J$ be a subset of the Cartesian product $\mathbb{N}_{N} \times \mathbb{N}_{N}$ and let $\mathcal{S}(J)$ be the pattern subspace defined by $J$ for the basis $\left\{e_{i}\right\}$. Every projection invariant for the algebra $A(J)$ has the form $P \oplus Q$, where $P$ and $Q$ are diagonal projections. If $Q=\operatorname{span}\left\{e_{k}: k \in K\right\}$ is a fixed diagonal projection, then, in order for $P \oplus Q$ to be invariant for $A(J)$, it is necessary and sufficient that $P \supseteq \bigvee\left\{e_{i}: i \in \bigcup\left\{J_{k}: k \in K\right\}\right\}$.

In case $Q=0$, then the span mentioned in the theorem is taken over an empty index set, and, in this case, we adopt the convention that the span is the zero subspace.

To make the theorem more clear, we now specialize to two examples.

Example 1. Let $\mathcal{H}$ be $N$-dimensional, with $N<\infty$, and let $J=\mathbb{N}_{N} \times \mathbb{N}_{N}$. Operators in $A(J)$ have the form

$$
\left[\begin{array}{cc}
D_{1} & S \\
0 & D_{2}
\end{array}\right]
$$

where $S$ can be any operator in $B(\mathcal{H})$. In this case, $J_{k}=\{1,2, \ldots, N\}$ for each $k$ and therefore, if $P \oplus Q$ is to be invariant for $A(J)$, we have two possibilities. First, if $Q=0$, then $P \supseteq\{0\}$, which is, of course, no condition at all; therefore, any projection of the form $P \oplus 0$ can lie in the lattice of invariant subspaces. Second, if $Q \neq 0$, then $J_{k}=\mathbb{N}_{N}$ and, consequently, any suitable $P$ must contain the span of all $e_{i}$; that is, we must have $P=I$. Thus, in this case, the lattice of $A(J)$ must contain precisely those projections of the form

$$
\left[\begin{array}{ll}
P & 0 \\
0 & 0
\end{array}\right] \text { and } \quad\left[\begin{array}{ll}
I & 0 \\
0 & Q
\end{array}\right]
$$

where $P$ and $Q$ can be any diagonal projections.

Example 2. Let $N=\infty$ and let $J=\{(i, k): i \neq k\}$. In this case, the pattern subspace $\mathcal{S}(J)$ consists of matrices whose diagonal entries are all zero. If $Q=$ 0 , then, as before, any diagonal projection $P$ will satisfy the criterion that $P \oplus$ $Q \in \operatorname{Lat}(A(J))$. Now, for any $k$, we have $J_{k}=\{1,2, \ldots, k-1, k+1, \ldots, \infty\}$. Consequently, if $Q$ contains any two different basis vectors $e_{k}$ and $e_{j}$, then the union of the cross-sections $J_{j}$ and $J_{k}$ contains all indices, and any $P$ for which $P \oplus Q \in \operatorname{Lat}(A(J))$ must be the identity projection. However, if $Q$ is rank-one for instance, if $Q$ is the span of just the vector $e_{k}$ - then the minimal $P$ for which $P \oplus Q \in \operatorname{Lat}(A(J))$ is the span of all those $e_{i}$ such that $i \neq k$ - in other words, the orthogonal complement of $Q$. We have now identified the lattice of $A(J)$; it consists of all projections of the forms

$$
\left[\begin{array}{ll}
P & 0 \\
0 & 0
\end{array}\right], \quad\left[\begin{array}{ll}
I & 0 \\
0 & Q
\end{array}\right] \text {, and }\left[\begin{array}{cc}
R^{\perp} & 0 \\
0 & R
\end{array}\right]
$$

for any diagonal projections $P$ and $Q$ and any rank-one diagonal projection $R$. 
We now return to a more general setting for some remarks. Let $\mathcal{L}$ be a commutative subspace lattice and let $\operatorname{Alg} \mathcal{L}$ be its associated CSL algebra. Let $X$ and $Y$ be operators in $B(\mathcal{H})$ (not necessarily in $A \lg \mathcal{L}$ ) and suppose that there is an operator $A$ in $A \lg \mathcal{L}$ such that $A X=Y$. For any $E \in \mathcal{L}$, we have $E^{\perp} A E^{\perp}=A E^{\perp}$, and so, for any vector $f$,

$$
\begin{aligned}
A X & =Y, \\
E^{\perp} A X & =E^{\perp} Y, \\
E^{\perp} A E^{\perp} X & =E^{\perp} Y, \\
E^{\perp} A E^{\perp} X f & =E^{\perp} Y f,
\end{aligned}
$$

and consequently we have, for all $E \in \mathcal{L}$ and all $f \in \mathcal{H}$,

$$
\left\|E^{\perp} Y f\right\| \leq\left\|E^{\perp} A\right\|\left\|E^{\perp} X f\right\| \leq\|A\|\left\|E^{\perp} X f\right\| .
$$

So we have

$$
\sup \left\{\frac{\left\|E^{\perp} Y f\right\|}{\left\|E^{\perp} X f\right\|}: E \in \mathcal{L} \text { and } f \in \mathcal{H}\right\} \leq\|A\|
$$

We follow the convention that any fraction of the form $0 / 0$ that appears in this supremum must be evaluated as 0 . Therefore, in order that there exist an operator $A$ in $A \lg \mathcal{L}$ such that $A X=Y$, it is necessary that the supremum in the display above be finite; and, furthermore, that supremum provides a lower bound for the norm of any such $A$. It is a result of Hopenwasser that, if $X$ is rank-one, then the condition is also sufficient. We state this as a theorem, in a slightly altered form:

Theorem (Hopenwasser). Let $\mathcal{L}$ be a commutative subspace lattice in $B(\mathcal{H})$ and let $X$ be an operator of rank 0 or 1 acting on $\mathcal{H}$, and let $Y \in B(\mathcal{H})$. The following conditions are equivalent:

(1) There exists an operator $A$ in Alg $\mathcal{L}$ such that $A X=Y$.

(2) $\sup \left\{\frac{\left\|E^{\perp} Y f\right\|}{\left\|E^{\perp} X f\right\|}: E \in \mathcal{L}\right.$ and $\left.f \in \mathcal{H}\right\} \leq \infty$.

Furthermore, if condition (2) holds, then there is an operator $A \in A \lg \mathcal{L}$ such that $A X=Y$ and whose norm is equal to the supremum in (2).

The case where the rank is zero is trivially true, since all of the fractions involved are $0 / 0$, but we have stated the theorem in this fashion in order to draw a parallel between the cases when $X$ has very small rank ( 0 or 1$)$ and very small co-rank. Indeed, if the co-rank of $X$ is 0 , then condition (2) is again sufficient, as we will now show. If the co-rank of $X$ is 0 , then the range of $X$ is dense. For ease of reference, we adopt the notation $\rho(X, Y ; \mathcal{L})$ for the supremum in condition $(2)$.

Theorem. Let $\mathcal{L}$ be a commutative subspace lattice in $B(\mathcal{H})$ and let $X$ be an operator with dense range, and let $Y \in B(\mathcal{H})$. The following conditions are equivalent:

(1) There exists an operator $A$ in $A$ lg $\mathcal{L}$ such that $A X=Y$.

(2) $\rho(X, Y ; \mathcal{L}) \stackrel{\text { def }}{=} \sup \left\{\left\|E^{\perp} Y f\right\| /\left\|E^{\perp} X f\right\|: E \in \mathcal{L}\right.$ and $\left.f \in \mathcal{H}\right\}<\infty$.

Furthermore, if condition (2) holds, then there is an operator $A \in \operatorname{Alg} \mathcal{L}$ such that $A X=Y$ and whose norm is equal to $\rho(X, Y ; \mathcal{L})$.

Proof. Let $\rho(X, Y ; \mathcal{L})=K$. Condition $(2)$ is equivalent to the condition $Y^{*} E^{\perp} Y \leq$ $K^{2} X^{*} E^{\perp} X$, for all $E \in \mathcal{L}$. In particular, $Y^{*} Y \leq K^{2} X^{*} X$, and, by Douglas' range inclusion theorem [1], we know that there is an operator $D$ of norm no greater than 
$K$ such that $D X=Y$. In fact, by the remarks preceding Hopenwasser's theorem, the norm of $D$ can be no smaller than $K$ either, so $\|D\|=K$. Because the range of $X$ is dense, the operator $D$ is unique and we need to prove only that $D$ lies in $\operatorname{Alg} \mathcal{L}$. Since $\left\|E^{\perp} Y f\right\| \leq K\left\|E^{\perp} X f\right\|$ and $D X=Y$, we have $\left\|E^{\perp} D X f\right\| \leq K\left\|E^{\perp} X f\right\|$ for all $E$ and $f$; since $D$ is bounded and the range of $X$ is dense, we have $\left\|E^{\perp} D g\right\| \leq$ $K\left\|E^{\perp} g\right\|$ for all $g \in \mathcal{H}$. If $g \in E$, then $E^{\perp} D g=0$, that is, $D g$ lies in $E$ as well. Thus, $E$ is invariant for $D$ and we are done.

Thus, if $X$ has either rank 0 or co-rank 0 , condition (2) is both necessary and sufficient for the existence of an operator $A \in A \lg \mathcal{L}$ such that $A X=Y$. Of course, Hopenwasser's result is that the same is true for $X$ of rank 1 . What is the case if $X$ has co-rank 1? Can we find, in this case, an interpolating operator $A$ ? There is an example in [4] which shows that - even when the dimension of $\mathcal{H}$ is 3 we may not be able to do this if we insist that the norm of $A$ be no greater than $\rho(X, Y ; \mathcal{L})$. Briefly, the example is this: The operators act on the Hilbert space $\mathbb{C}^{3}$ and the equation $A X=Y$ has the form

$$
\left[\begin{array}{ccc}
* & 0 & * \\
0 & * & * \\
0 & 0 & *
\end{array}\right]\left[\begin{array}{ccc}
0 & \sqrt{2} & 1 \\
0 & 1 & 0 \\
0 & 0 & 1
\end{array}\right]=\left[\begin{array}{lll}
0 & 1 & 0 \\
0 & 0 & 1 \\
0 & 0 & 0
\end{array}\right]
$$

where the $*$ 's represent entries that are free to be chosen. In this case, $\rho(X, Y ; \mathcal{L})=$ 1 but the only operator of the required form is

$$
\left[\begin{array}{ccc}
1 / \sqrt{2} & 0 & -1 / \sqrt{2} \\
0 & 0 & 1 \\
0 & 0 & 0
\end{array}\right]
$$

whose norm is obviously greater than 1 . A further example in that paper shows that we may have $\rho(X, Y ; \mathcal{L})=1$ but there may be no bounded operator $A$ in $A \lg \mathcal{L}$ at all that solves the equation $A X=Y$. However, that example is constructed from the basic one above in such a way that the rank and co-rank of the resulting $X$ are both infinite. Is it possible that, if the co-rank of $X$ is 1 , we will always be able to solve $A X=Y$, possibly with some $A$ of norm larger than $\rho(X, Y ; \mathcal{L})$ ? We intend to show that this is not the case. Note, however, that, if the underlying space is finite-dimensional, then a simple application (which we omit) of the Hahn-Banach Theorem shows the following:

Theorem. Let $\mathcal{H}$ be a Hilbert space of dimension $n<\infty$. If $\mathcal{L}$ is a commutative subspace lattice acting on $\mathcal{H}$ and if $X$ and $Y$ are operators such that $\rho(X, Y ; \mathcal{L})<\infty$, then there is an operator $A$ in Alg $\mathcal{L}$ such that $A X=Y$ and such that $\|A\| \leq$ $n \cdot \rho(X, Y ; \mathcal{L})$.

The factor $n$ can actually be made a bit smaller, but in any case the theorem is not very satisfactory, since the factor increases with the size of the space. Necessarily, then, our counterexample must operate on an infinite-dimensional space. We want to construct a lattice $\mathcal{L}$ acting on an infinite-dimensional space and operators $X$ and $Y$ such that the co-rank of $X$ is 1 and $\rho(X, Y ; \mathcal{L})=1$ but such that there is no bounded operator $A \in A \lg \mathcal{L}$ such that $A X=Y$.

Example. We use the lattice of Example 2, and assume that the Hilbert space is separable and infinite-dimensional, with fixed basis $\left\{e_{1}, e_{2}, \ldots\right\}$. The projections 
in the lattice have the forms

$$
E=\left[\begin{array}{ll}
I & 0 \\
0 & Q
\end{array}\right], \quad\left[\begin{array}{cc}
P & 0 \\
0 & 0
\end{array}\right], \text { and } \quad\left[\begin{array}{cc}
R^{\perp} & 0 \\
0 & R
\end{array}\right]
$$

and so the orthogonal complements have the forms

$$
E^{\perp}=\left[\begin{array}{ll}
0 & 0 \\
0 & Q
\end{array}\right], \quad\left[\begin{array}{cc}
P & 0 \\
0 & I
\end{array}\right] \text {, and } \quad\left[\begin{array}{cc}
R & 0 \\
0 & R^{\perp}
\end{array}\right]
$$

where, as before, $P$ and $Q$ represent any diagonal projections and $R$ represents a rank-one diagonal projection. (If $P$ is diagonal, so is $P^{\perp}$, so, for simplicity, we have dropped the " $\perp$ " symbol.) The condition $\rho(X, Y ; \mathcal{L})=1$ is the same as requiring that $Y^{*} E^{\perp} Y \leq X^{*} E^{\perp} X$ for all $E \in \mathcal{L}$. Rather than tip our hand by presenting a particular choice of $X$ and $Y$ at the outset, we will reveal the shape of the operators gradually. First, we choose $X$ and $Y$ to have the form

$$
X=\left[\begin{array}{cc}
I & 0 \\
0 & X_{1}
\end{array}\right] \text { and } Y=\left[\begin{array}{cc}
0 & Y_{1} \\
0 & 0
\end{array}\right]
$$

so that the condition $A X=Y$ for $A \in A(J)$ is the same as the equation $B X_{1}=Y_{1}$, where $B$ is the northeast corner of $A$ and we may without harm assume that the other entries of $A$ are 0 . Note that $X$ and $X_{1}$ have the same co-rank. The conditions $Y^{*} E^{\perp} Y \leq X^{*} E^{\perp} X$ are automatic in case $E^{\perp}$ has the first form listed above. For the second form, the conditions reduce to the inequality $Y_{1}^{*} P Y_{1} \leq X_{1}^{*} X_{1}$. Since $Y_{1}^{*} P Y_{1} \leq Y_{1}^{*} Y_{1}$ for any projection $P$, all of these conditions collapse to the assumption that $Y_{1}^{*} Y_{1} \leq X_{1}^{*} X_{1}$. It remains to consider the condition $Y^{*} E^{\perp} Y \leq$ $X^{*} E^{\perp} X$ when $E$ has the form $R^{\perp} \oplus R$ for a rank-one diagonal projection $R$. In this case, the inequality reduces to $Y_{1}^{*} R Y_{1} \leq X_{1}^{*} R^{\perp} X_{1}$. We now make the further assumption that $X_{1}$ is an orthogonal projection - however, $X_{1}$ will not be diagonal in the basis $\left\{e_{n}\right\}$ - and that $Y_{1}=D X_{1}$, where $D$ is a self-adjoint diagonal operator of norm 1 to be specified later; for now we simply let the diagonal entries of $D$ be called $\left\{d_{j}\right\}$, and we let $R_{j}=e_{j} \otimes e_{j}{ }^{*}$. The condition $Y_{1}^{*} R_{j} Y_{1} \leq X_{1}^{*} R_{j}^{\perp} X_{1}$ becomes

$$
\begin{aligned}
X_{1} D R_{j} D X_{1} & \leq X_{1} X_{1}-X_{1} R_{j} X_{1} \\
\text { or } \quad X_{1}\left(R_{j}+D R_{j} D\right) X_{1} & \leq X_{1}^{2} \\
\text { and so } \quad X_{1}\left(1+\left|d_{j}\right|^{2}\right) R_{j} X_{1} & \leq X_{1} X_{1}
\end{aligned}
$$

because $D$ and $R$ are both diagonal and $D R D=D\left(e_{j} \otimes e_{j}^{*}\right) D=\left|d_{j}\right|^{2} e_{j} \otimes e_{j}^{*}$. We now have the requirement that, for all $j$,

$$
X_{1} R_{j} X_{1} \leq \frac{1}{1+\left|d_{j}\right|^{2}} X_{1}^{2}
$$

which is equivalent to the condition $\left\|R_{j} X_{1} f\right\|^{2} \leq\left(1+\left|d_{j}\right|^{2}\right)^{-1}\left\|X_{1} f\right\|^{2}$, for any vector $f$. Thus, with $R=e_{j} \otimes e_{j}^{*}$,

$$
\begin{aligned}
\left|\left\langle f, X_{1} e_{j}\right\rangle\right|^{2} & =\left|\left\langle X_{1} f, e_{j}\right\rangle\right|^{2} \\
& =\left\|R X_{1} f\right\|^{2} \\
& \leq \frac{1}{1+\left|d_{j}\right|^{2}}\left\|X_{1} f\right\|^{2} \\
& \leq \frac{1}{1+\left|d_{j}\right|^{2}}\|f\|^{2}
\end{aligned}
$$


and, since this inequality holds for all $f$, we know that $\left\|X_{1} e_{j}\right\|^{2} \leq\left(1+\left|d_{j}\right|^{2}\right)^{-1}$. On the other hand, if $\left\|X_{1} e_{j}\right\|^{2} \leq\left(1+\left|d_{j}\right|^{2}\right)^{-1}$, then it is easy to see that $\left\|R_{j} X_{1} f\right\|^{2} \leq$ $\left(1+\left|d_{j}\right|^{2}\right)^{-1}\left\|X_{1} f\right\|^{2}$. Thus, the condition $Y^{*} E^{\perp} Y \leq X^{*} E^{\perp} X$ is equivalent to the requirement that $\left\|X_{1} e_{j}\right\|^{2} \leq\left(1+\left|d_{j}\right|^{2}\right)^{-1}$ for all $j$. Letting $Q$ represent the projection onto the orthogonal complement of the range of $X$, namely, $Q=I-X$, we have $\left\|Q e_{j}\right\|^{2}=\left\|e_{j}\right\|^{2}-\left\|X e_{j}\right\|^{2} \geq\left|d_{j}\right|^{2}\left(1+\left|d_{j}\right|^{2}\right)^{-1}$. Finally, we restrict to the case when $Q$ has rank one; if $Q=h \otimes h^{*}$, then $\left\|Q e_{j}\right\|=\left|\left\langle h, e_{j}\right\rangle\right|$; for simplicity, let $\left\langle h, e_{j}\right\rangle$ be denoted by $h_{j}$. Since - if $Q$ is to be a projection - we must have $\|h\|=1$, it is necessary that

$$
\sum_{j=1}^{\infty} \frac{\left|d_{j}\right|^{2}}{1+\left|d_{j}\right|^{2}} \leq 1 .
$$

On the other hand, suppose that there is an operator $B$ in $\mathcal{S}(J)$ such that $B X_{1}=Y_{1}$. Then $B$ and $D$ must agree on the range of $X$; that is, $B$ and $D$ can differ only by a rank-one operator of the form $x \otimes h^{*}$. Let $B=D+x \otimes h^{*}$ and observe that the requirement $\left\langle B e_{j}, e_{j}\right\rangle=0$ means that $0=d_{j}+x_{j} h_{j}$, where we use the notation $x_{j}=\left\langle x, e_{j}\right\rangle$. Consequently, $x_{j}=-d_{j} / h_{j}$, and, in order that $x$ have finite norm, it would be necessary that

$$
\sum \frac{\left|d_{j}\right|^{2}}{\left|h_{j}\right|^{2}}<\infty .
$$

We are now ready, at last, to present a particular example. In order to specify $X$ and $Y$, we only need to specify the vector $h$ and the diagonal operator $D$. We want condition (*) to hold, along with the requirement that $\left|h_{j}\right|^{2} \geq\left|d_{j}\right|^{2}\left(1+\left|d_{j}\right|^{2}\right)^{-1}$; but we want condition $(* *)$ to fail. For a simple specific instance, let $d_{j}=\left(2^{j}-1\right)^{-1 / 2}$ and let $h_{j}=d_{j}\left(1+d_{j}^{2}\right)^{-1 / 2}$. Then, $h_{j}^{2}=2^{-j}$ and so

$$
\sum_{j=1}^{\infty} h_{j}^{2}=\sum_{j=1}^{\infty} \frac{1}{2^{j}}=1 .
$$

But

$$
\sum \frac{d_{j}^{2}}{h_{j}^{2}}=\sum\left(1+d_{j}^{2}\right)=\infty .
$$

The example is complete.

Actually, if $h$ is any unit vector with infinitely many non-zero components $h_{j}$, we can find an operator $D$ that provides a counterexample, since the function $x \mapsto x^{2}\left(1+x^{2}\right)^{-1}$ maps the interval $[0, \infty)$ invertibly onto the interval $[0,1)$. What this means is that, for any projection $X$ whose co-range is spanned by a single vector $h$ which has infinitely many non-zero components (with respect to the basis vectors), we can find an operator $Y$ such that $\rho(X, Y ; \mathcal{L})=1$ but so that there is no bounded operator $A \in A(J)$ such that $A X=Y$.

\section{REFERENCES}

1. R. G. Douglas, On Majorization, Factorization, and Range Inclusion of Operators on Hilbert Space, Proc. Amer. Math. Soc. 17 (1966), 413-415. MR 34:3315

2. E. Katsoulis, R. Moore, and T. Trent, Interpolation in Nest Algebras and Applications to Operator Corona Theorems, Journal of Operator Theory 29 (1993), 115-123. MR 95b:47052 
3. R. Moore and T. Trent, Solving Operator Equations in Nest Algebras, Houston Journal of Mathematics, to appear.

4. R. Moore, and T. Trent, Interpolation in Inflated Hilbert Spaces, Proc. Amer. Math. Soc. Journal of Operator Theory, to appear. CMP 98:01

Department of Mathematics, University of Alabama, Tuscaloosa, Alabama 35487 0350 